\title{
Ventriculectomia Parcial Esquerda. Resultados Atuais, Possíveis Indicações e Perspectivas Futuras
}

\author{
Reinaldo B. Bestetti, José Carlos F. Brasil, Rubio Bombonato \\ Ribeirão Preto, SP
}

Em 1995, Batista e col ${ }^{1}$ descreveram nova técnica cirúrgica para o tratamento de portadores de insuficiência cardíaca congestiva (ICC) terminal, originalmente denominada ventriculectomia parcial. A técnica consiste basicamente na retirada de um fragmento da parede lateral do ventrículo esquerdo (VE), do ápice ventricular ao anel da válvula mitral, através dos músculos papilares, sob circulação extracorpórea, sem o uso de cardioplegia. Os resultados obtidos no pós-operatório imediato de 18 pacientes submetidos a esta nova técnica operatória mostraram aumento na fração de ejeção do VE(FEVE) de 100 a 300\%.

Apesar de ostensivamente divulgada na imprensa leiga, tanto no Brasil como no exterior ${ }^{2}$, poucas são as publicações científicas referentes à ventriculectomia parcial. Devido a isso, apenas alguns grupos no Brasil ${ }^{2,3}$ e no exterior ${ }^{5-7}$ têm, sistematicamente, realizado este procedimento cirúrgico para avaliar sua potencial eficácia como tratamento alternativo para pacientes selecionados com ICC terminal.

Embora os resultados iniciais sejam alentadores, existem incertezas com relação ao prognóstico a longo prazo do procedimento, não sendo ainda claras, conseqüentemente, as diretrizes para a realização da ventriculectomia parcial.

Em que pese o surgimento dos antagonistas da enzima de conversão do angiotensinogênio em angiotensina, que melhorou a sobrevida de pacientes com ICC, a mortalidade anual continua a ser alta (>40\%) naqueles com ICC avançada, ou seja, grau IV da classificação funcional da New York Heart Association (NYHA) ${ }^{8,9}$, FEVE $<0,20$ determinada pela ventriculografia isotópica ${ }^{9,10}$, diâmetro diastólico do VE (DDVE) $>75 \mathrm{~mm}^{11,12}$, caminhada em $6 \mathrm{~min}<300 \mathrm{~m}^{13}$, consumo máximo de oxigênio durante exercício aeróbico $<13$ mets ${ }^{10,14}$, percentagem de encurtamento da fibra miocárdica $<13 \% \mathrm{e}$ FEVE $<0,30$ determinados ecocardiograficamente ${ }^{15-17}$ eíndice cardíaco $<2,51 / \mathrm{min} / \mathrm{m}^{2}$ ou pressão capilar pulmonar $>15 \mathrm{mmHg}{ }^{9,18}$. Nesses pacientes, o transplante cardíaco ortotópico pode ser a única opção para se melhorar a qualidade de vida e a sobrevida. Entretanto, o transplante cardí-

Hospital do Coração de Ribeirão Preto

Correspondência: Reinaldo B. Bestetti - Serviço de Saúde-PCARP - Av. Bandeirantes, 3900 - 14049-900 - Ribeirão Preto, SP

Recebido para publicação em 13/6/97

Aceito em 10/9/97 aco constitui tratamento caro e complexo, limitando sua realização a apenas alguns centros de nosso país. Assim, o surgimento de alternativa terapêutica para o tratamento de pacientes com ICC terminal é altamente desejável.

\section{Resultados atuais da ventriculectomia parcial}

Pós-operatório imediato (primeiros 30 dias) - Até o momento, ao que parece, existem dados científicos consistentes referentes a 84 pacientes submetidos à ventriculectomia parcial na literatura médica. Os dados relativos ao pós-operatório (PO) imediato são conhecidos em 73 (86\%) pacientes. Bellotti e $\mathrm{col}^{4}$ estudaram 11 pacientes $17 \pm 4$ dias após a ventriculectomia parcial, verificando importante regressão na classe funcional (CF) da NYHA $(3,6 \pm 0,5 \mathrm{x}$ $1,4 \pm 0,7, p<0,001)$, eno DDVE $(80 \pm 7 \times 71 \pm 7 \mathrm{~mm}, \mathrm{p}=0,002)$, aumento na percentagem de encurtamento da fibra miocárdica $(11,5 \pm 1,8 \times 19,8 \pm 3,9, \mathrm{p}<0,001)$ e alteração na geometria do VE, que se tornou mais hipertrófico e menos esférico. Kirshner e col $^{6}$ observaram melhora na CF da NYHA (3,9 para $2,7, \mathrm{p}=0,01$ ), diminuição do DDVE (69 para 58mm, $\mathrm{p}=0,006)$ e aumento da $\operatorname{FEVE}(0,21$ para $0,34, \mathrm{p}=0,03) 26$ dias após a ventriculectomia parcial. Starling e $\mathrm{col}^{5}$ verificaram aumento daFEVE $(0,15 \pm 0,07 \times 0,37 \pm 0,11, \mathrm{p}<0,0001)$ e diminuiçãodoDDVE $(80 \pm 11 \times 59 \pm 6 \mathrm{~mm}, \mathrm{p}<0,0001)$ imediatamente após a cirurgia. Aumento médio da FEVE $(0,17$ para 0,31 , $\mathrm{p}<0,01)$ e diminuição na CF da NYHA $(3,7$ para 2,6, $\mathrm{p}<0,01)$ também foram relatados por Takeshita e $\mathrm{col}^{7} 9 \pm 6$ dias após a ventriculectomia parcial. Starling e $\mathrm{col}^{5}$ detectaram aumento no índice cardíaco $\left(2,0 \pm 0,6 \times 2,6 \pm 0,6 \mathrm{ml} / \mathrm{min} / \mathrm{m}^{2}\right.$, $\mathrm{p}=0,01)$ e diminuição na pressão atrial esquerda $(24 \pm 12 \mathrm{x}$ $13 \pm 4 \mathrm{mmHg}, \mathrm{p}=0,0006$ ) imediatamente após o procedimento cirúrgico. Estudo hemodinâmico realizado por Takeshita e $\operatorname{col}^{7} 9$ dias em média após a ventriculectomia também mostrou aumento do índice cardíaco (4,35 para $5,48 \mathrm{ml} / \mathrm{min} / \mathrm{m}^{2}$, $\mathrm{p}<0,01)$.

Assim, os dados disponíveis na literatura sugerem que a redução cirúrgica da cavidade ventricular acompanha-se de melhora na forma geométrica e no desempenho do VE, diminuição da congestão pulmonar secundária à disfunção sistólica e melhora na sintomatologia logo após o procedimento.

A mortalidade no PO imediato parece ser razoável. Batista e col ${ }^{19}$ relataram a ocorrência de óbito em 13(12\%) pacientes nesse período, mas as características clínicas, prin- 
cipalmente a estabilidade hemodinâmica, não foram descritas em detalhes. Observamos ${ }^{20}$ óbito em três pacientes operados em choque cardiogênico após o sucesso obtido com os sete primeiros pacientes. Contudo, deve-se enfatizar que não houve óbito nas séries em que os pacientes estavam estáveis, do ponto de vista hemodinâmico, quando da realização da cirurgia.

A morbidade no PO imediato da ventriculectomia também parece ser baixa. Bombonato e $\mathrm{col}^{3}$ relataram a ocorrência de taquicardia ventricular, enquanto que Starling e col ${ }^{21}$ descreveram dois episódios de falência ventricular, que necessitaram de assistência ventricular mecânica, em 2 (11\%) pacientes submetidos à ventriculectomia. Contudo, esses pacientes talvez não tenham sido semelhantes aos outros descritos em outras séries, pois um deles teve infarto agudo do miocárdio (IAM) no período peri-operatório, e, o outro, IAM não detectado antes do procedimento cirúrgico.

Pós-operatório tardio (30 a 120 dias após o procedimento) - Resultados após o PO imediato são disponíveis em $12(14 \%)$ pacientes. Bombonato e col $^{3}$ observaram em 7 casos melhora naFEVE $(0,15 \pm 0,05 \times 0,22 \pm 0,04, \mathrm{p}=0,02)$, na CF da NYHA $(4,0 \pm 0 \times 1,71 \pm 0,48, \mathrm{p}=0,009)$ e no DDVE $(75,67 \pm 11,98 \times 64,67 \pm 11,41 \mathrm{~mm}, \mathrm{p}=0,02) 60$ dias após a ventriculectomia. Achados semelhantes foram relatados por Takeshita e $\mathrm{col}^{7}$, posteriormente: aumento na FEVE $(17,4$ para $30, \mathrm{p}<0,05)$ e diminuição na $\mathrm{CF}$ da NYHA $(3,7$ para 2,2, p<0,05) em cinco pacientes 136 195 dias após o ato operatório. Portanto, verifica-se que a melhora no desempenho do VE induzido pela redução da cavidade ventricular, traduzida clinicamente pela melhora na CF observada no PO imediato, mantém-se dois a quatro meses após a cirurgia. É importante frisar que não houve morbidade nos dois trabalhos citados.

Longo prazo (>1 ano após o procedimento) - Apenas um grupo relatou, até o momento, sua experiência a longo prazo com sete $(8 \%)$ pacientes submetidos à ventriculectomia $^{22}$. Dos sete pacientes operados, consecutivamente, $\operatorname{cinco}(71 \%)$ sobreviveram, sendo que um faleceu de hemorragia digestiva, e um outro, que havia abandonado o tratamento por conta própria, subitamente. Quatro dos sete $(57 \%)$ pacientes foram internados para compensação de ICC e todos não estavam recebendo o tratamento preconizado pelo grupo, seja por conta própria, seja por orientação do médico de origem. Um (14\%) caso, que não tinha trombos no ecocardiograma bidimensional, apresentou acidente vascular cerebral embólico.

Um ano após a ventriculectomia, os autores observaram melhora na $\mathrm{CF}(4,0 \pm 0 \times 1,40 \pm 0.54, \mathrm{p}=0,003)$, no DDVE $(73,20 \pm 11,21 \times 64,20 \pm 9,33 \mathrm{~mm}, \mathrm{p}=0,003)$ e naFEVEdeterminada ecocardiograficamente $(0,29 \pm 0,07 \times 0,48 \pm 0,10, p=0,02)$ nos pacientes sobreviventes. Admite-se, portanto, que a melhora na CF e no desempenho ventricular, observados logo após o procedimento, mantêm-se um ano após a ventriculectomia.

\section{Possíveis indicações para a ventriculectomia}

O procedimento deve ficar reservado para pacientes que estão ou que estiveram recentemente no grau IV da classificação da NYHA, pois, pacientes que atingiram, no máximo, o grau III têm baixa mortalidade anual $(20 \%)^{23}$. Os pacientes em grau IV, que necessitam de suporte inotrópico, os que apresentam o pior prognóstico a curto prazo $^{24}$, porém estabilizados do ponto de vista hemodinâmico, têm sido operados com sucesso ${ }^{3}$, mas o mesmo parece não acontecer com aqueles que apresentam instabilidade hemodinâmica ${ }^{20}$. Conseqüentemente, o procedimento deve ser indicado para pacientes em grau IV, estabilizados hemodinamicamente.

A cardiopatia chagásica crônica, quando comparada a outras etiologias de ICC, apresenta pior prognóstico ${ }^{25}$. Pacientes com ICC secundária a diferentes etiologias, incluindo a chagásica, têm sido operados com sucesso. Portanto, até que surjam evidências em contrário, a etiologia da ICC não deve ser levada em conta na indicação do procedimento cirúrgico.

Observa-se, um ano após a ventriculectomia, redução de aproximadamente $15 \%$ no DDVE. Em assim sendo, a ventriculectomia poderia ser realizada idealmente em pacientes com DDVE variando de 75 a 90mm, pois dimensões da cavidade ventricular $<75 \mathrm{~mm}$ associam-se a bom prognóstico, enquanto que valores $>90 \mathrm{~mm}$ ainda manteriam o DDVE em níveis associados a mau prognóstico ${ }^{12}$.

A deambulação de um indivíduo com ICC em estágio avançado $<300 \mathrm{~m}$ durante $6 \mathrm{~min}$ consecutivos (teste dos $6 \mathrm{~min}$ ) é importante índice prognóstico a curto prazo. Levando-se em consideração as facilidades para a realização do teste dos 6min, parece razoável a utilização deste teste na indicação do procedimento, de tal forma que indivíduos que percorram $<300 \mathrm{~m}$ poderiam ser submetidos à ventriculectomia.

Uma vez que os valores percentuais de encurtamento da fibra $<13$ e da FEVE $<0,30$ estão relacionados a alta taxa de mortalidade anual ${ }^{15-17}$, esses indicadores prognósticos devem ser levados em conta na indicação do procedimento. Como os resultados a longo prazo mostram aumento de $50 \mathrm{a}$ $60 \%$ nos valores de FEVE, obtidos pela ecocardiografia ou ventriculografia isotópica ${ }^{22}$, percebe-se que portadores de FEVE extremamente baixos podem não se beneficiar do procedimento. Conseqüentemente, valores de FEVE $<0,15$ na ventriculografia isotópica e $<0,19$ na ecocardiografia talvez sejam limítrofes para a realização da ventriculectomia.

A tabela I resume as possíveis indicações para a realização da ventriculectomia parcial.

\section{Perspectivas}

Com base nos resultados obtidos até agora, pode-se dizer que a ventriculectomia parcial esquerda, em pacientes selecionados, parece ser útil no tratamento da ICC em estágio avançado. Em nosso meio, os pacientes que provavelmente mais possam ser beneficiados pelo procedimento são 
Tabela I - Possíveis indicações para a realização da ventriculectomia parcial esquerda

1. Grau IV da NYHA

2. $90>$ DDVE $>75 \mathrm{~mm}$

3. FEVE $<0,20$ na VI

4. FEVE $<0,30, \% \mathrm{EF}<13$ no ECO

5. Deambulação $<300 \mathrm{~m}$ em $6 \mathrm{~min}$

NYHA- New York Heart Association; DDVE- diâmetro diastólico do ventrículo esquerdo; FEVE- fração de ejeção do ventrículo esquerdo; VI- ventriculografia radioisotópica; ECO- ecocardiograma bidimensional; EF- encurtamento da fibra miocárdica. aqueles que apresentam DDVE entre 75 e $90 \mathrm{~mm}$, FEVEentre 0,19 e 0,30 ao ecocardiograma, deambulação em $6 \mathrm{~min}<300 \mathrm{~m}$ e grau IV da NYHA estabilizados hemodinamicamente. Portanto, quando não se vislumbra a possibilidade de realizar transplante cardíaco em pacientes com ICC terminal, tratados clinicamente de forma adequada, a ventriculectomia pode estar indicada. Entretanto, a fim de se ter certeza do benefício da ventriculectomia na sobrevida de pacientes com ICC em estágio avançado, os dados até agora disponíveis apontam para a necessidade da realização de estudo prospectivo, duplo-cego, comparando-se a ventriculectomia com o tratamento clínico otimizado.

\section{Referências}

1. Batista RJV, Santos JLV, Cunha MA et al - Ventriculectomia parcial: um novo conceito no tratamento cirúrgico de cardiopatias em fase final. In: Anais do XXII Congresso Nacional de Cirurgia Cardíaca. Brasília: Sociedade Brasileira de Cirurgia Cardíaca, 1995: 150-1.

2. McCarthy M - Batista procedure proves its value in the USA. Lancet 1997; 349: 855 .

3. Bombonato R, Bestetti RB, Sgarbieri R et al - Experiência inicial com a ventriculectomia parcial esquerda no tratamento da insuficiência cardíaca terminal. Arq Bras Cardiol 1996; 66: 189-92.

4. Bellotti G, Moraes A, Bocchi EA et al - Efeitos da ventriculectomia parcial nas propriedades mecânicas, forma e geometria do ventrículo esquerdo em portadores de cardiomiopatia dilatada. Arq Bras Cardiol 1996; 67: 395-400.

5. Starling RC, Young JB, Scalia GM et al - Preliminary observations with ventricular remodeling surgery for refractory congestive heart failure (abstract). J Am Coll Cardiol 1997; 710.4: 64A.

6. Kirshner RL, Knight PA, Thompson MA et al - Ventricular reduction surgery as a treatment for idiopathic dilated cardiomyopathy. J Am Coll Cardiol 1997; 466A: 1064-169.

7. Takeshita N, Kawaguchi AT, Lima PRN et al-Hemodynamic changes in patients undergoing left ventricular diameter reduction (Batista operation) (abstract). J Am Coll Cardiol 1997; 710.3, 64A.

8. Fonarow GC, Fallick CC, Stevenson LW et al - Effect of direct vasodilation with hydralazine versus angiotensin converting enzyme inhibition with captopril on mortality in advanced heart failure: the HY-C trial. J Am Coll Cardiol 1992; 19: 842-50.

9. Keogh AM, Baron DW, Hicckie JB - Prognostic guides in patients with idiopathic or ischemic dilated cardiomyopathy assessed for cardiac transplantation. Am J Cardiol 1990; 65: 903-8.

10. Likoff MJ, Chandler SL, Kay HR - Clinical determinants of mortality in chronic congestive heart failure secondary to idiopathic dilated or ischemic cardiomyopathy. Am J Cardiol 1987; 59: 634-8.

11. Douglas PS, Morrow R, Ioli A, Reichek N - Left ventricular shape, afterload and survival in idiopathic dilated cardiomyopathy. J Am Coll Cardiol 1992; 13: 311-15.

12. Lee TH, Hamilton MA, Stevenson LW et al - Impact of left ventricular cavity size on survival in advanced heart failure. Am J Cardiol 1993; 72: 672-6.

13. Cahalin LP, Mathier MA, Semigran MJ, Dec WG, DiSalvo TG - The 6-minute walk test predicts peak oxygen uptake and survival in patients with advanced heart failure. Chest 1996; 110: 325-32.

14. Mancini DM, Eisen H, Kussmaul W, Mull R, Edmunds JR LH, Wilson JR - Value of peak oxygen consumption for optimal timing of cardiac transplantation in ambulatory patients with heart failure. Circulation 1991; 83: 778-86.

15. Baker BJ, Leddy C, Galie N, Casebolt P, Franciosa JA - Predictive value of Mmode echocardiography in patients with congestive heart failure. Am Heart J 1986; 111: 697-702.

16. Bestetti RB, Dalbó CMR, Freitas OC, Teno LAC, Castilho OT, Oliveira JSM Noninvasive predictors of mortality for patients with Chagas' heart disease: a multivariate stepwise logistic regression study. Cardiology 1994; 84: 261-7.

17. Mady C, Cardoso RHA, Barretto ACP, da Luz PL, Bellotti G, Pileggi F- Survival and predictors of survival in patients with congestive heart failure due to Chagas' cardiomyopathy. Circulation 1994; 90: 3098-102.

18. Hofmann T, Meinertz T, Kasper W et al - Mode of death in idiophatic dilated cardiomyopathy: a multivariate analysis of prognostic determinants. Am Heart J 1988; 116: 1455-63.

19. Batista RJV, Santos JLV, Franzoni M et al - Ventriculectomia parcial: um novo conceito no tratamento cirúrgico de cardiopatias em fase final. Rev Soc Bras Cir Cardiovasc 1996; 11: 1-6.

20. Bestetti RB, Bombonato R, Brasil JCF-Partial ventriculectomy: a promising treatment for patients with end-stage congestive heart failure. Circulation (in press).

21. Starling RC, Young JB, Buda TM, Vargo RL, Smedira NG, McCarthy PM-Patient selection for ventricular remodeling surgery: evolving criteria and outcomes (abstract). J Am Coll Cardiol 1997; 977.160: 246A.

22. Bestetti RB, Bombonato R, Kato M et al - Evolução clínica de pacientes submetidos à ventriculectomia esquerda parcial. Arq Bras Cardiol 1997; 68: 397-400.

23. Hamptom JR, van Veldhuisen DJ, Kleber FXet al-Randomised study of effect of ibopamine on survival in patients with advanced severe heart failure. Lancet 1997; 349: 971-7.

24. Anguita M, Arizon JM, Bueno G et al - Clinical and hemodynamic predictors of survival in patients aged $<65$ years with severe congestive heart failure secondary to ischemic or nonischemic dilated cardiomyopathy. Am J Cardiol 1993; 72 : 413-17.

25. Bestetti RB, Muccillo G-Clinical course of Chagas' heart disease: a comparison with dilated cardiomyopathy. Int J Cardiol 1997; 60: 187-93. 